\title{
EDITORIAL
}

nature

cell biology

\section{Less is More}

\section{User-friendly technology has made for slick slide presen- tations - but has style come at the expense of substance?}

PowerPoint is a joy to use - click a few highly intuitive buttons to animate your favourite model and the results seem the work of professionals with years of film school behind them. Customize your graphics to rival the finest glossy magazines. But does a visually striking presentation really make your research more accessible or memorable? Do bouncing phosphates really explain a kinase reaction better? We would argue that overbearing graphics tend to distract the audience from the science.

The mobile ringtone craze seems to have been replaced by an addiction to fancy slide transitions which seem irresistible to the multiconference-hopping Principal Investigator stuck in an airport lounge waiting for an indeterminably delayed flight. The final frontier in the multimedia circus of some presentations seems to be audio - it is just a matter of time before the first experiment is enhanced with the opening bars of Beethoven's Fifth.

This journal will certainly not bemoan the advent of the digital age (although PowerPoint lacks the romanticism of a mildewed first few slides to say 'this is where it all started'). Neither does it intend to undervalue a bit of light entertainment to keep the audience's attention on the last day of an overloaded conference. More importantly, for the cell biologist, modern presentation technology has of course been tremendously beneficial; in particular the real-time presentation of sizeable movies and the spatial navigation of three-dimensional microscopy images or protein structures permit data-rich and accessible presentations.

However, graphics tools ought to be used only when necessary. It is worth reflecting on the frustrating experience of watching a Hollywood movie so overloaded with special effects that it leaves the viewer drained from sensory overload but intellectually and emotionally unsatisfied. Less is more: after a day of back-to-back talks, nothing is more refreshing than a visually clear, logically constructed and well articulated presentation.

A case in point was a recent involuntary but highly effective experiment by Daniel A. Haber of the Massachusetts General Hospital Cancer Center, who gave a presentation on the last day of the $\mathrm{CNIO}$ / Nature conference 'Oncogenes: the next 25 years' last month in Madrid. Daniel decided to travel light and relied on the pen drive in his right-hand jacket pocket for his slides. Unfortunately it was only during the pre-talk coffee break that he noticed his pen drive was not in any of his pockets. Utterly unperturbed, he presented an oratory masterpiece apparently covering all the data on his slides (which he occasionally tried to visualize with a laser pointer on the blank screen). Remarkably, he stayed precisely on time (one wonders if that would have been achievable with slides) and his findings were accessibly presented. Despite the late hour, the audience was well engaged and focused on the speaker - the house lights were up and the speaker faced his audience. Most would agree that this was one of the most memorable talks in an altogether excellent conference.
The physical sciences - which of course require the presentation of highly complex concepts - have retained their traditional use of minimal visual aids. Cell biology is an observation-based discipline, and this may explain the field's affinity for visual support. Nevertheless, some 'do without' by choice: Sydney Brenner has famously done away with slides altogether and certainly gives striking presentations. We would not necessarily advocate such a dramatic move - unless of course you can marshal Daniel Haber's undeniable oratory skills. However, it is worth taking a lesson from this experiment: focus audience attention on the speaker, do not read off the screen and reserve slides to present key data and to summarize a complex body of work. Why not introduce the talk without slides?

This journal predicts that a new minimalism in conference presentations will be de rigueur for the next conference season.

\section{Of Mice and Men}

\section{The mouse knockout Nobel serves to highlight the need to share the published reagents for this core technique.}

This year's Nobel prize in Physiology or Medicine - awarded to Oliver Smithies, Mario Capecchi and Martin Evans for '...introducing specific gene modifications in mice by the use of embryonic stem cells'was a well expected and richly deserved announcement. After all, the technique provides a cornerstone of many key findings by allowing them to be validated in a physiological setting. For the same reason, we would like to re-emphasize that new knockout models fall under our policy for the unrestricted distribution of materials and methods (see www.nature.com/authors/editorial_policies/availability and May 2006 editorial). It is expensive and legally cumbersome to transport live stock. However, the distribution of ES cells or sperm is certainly feasible and we strongly recommend using public repositories such as the Jackson Laboratory, the Mutant Mouse Regional Resource Centers and the Federation of International Mouse Resources.

It is understandable that scientists want to reap academic credit for their mouse knockouts. However, this must not result in blocking access to published reagents. Authorship on follow-on studies may be appropriate, but again note our explicit policies on authorship (www.nature.com/authors/editorial_policies/authorship and March 2005 editorial). If you do encounter a persistent refusal to comply with these guidelines please contact the editors.

Often it is not the researchers themselves but overzealous intellectual property offices that restrict material sharing through prohibitive MTAs. It is therefore important to keep your institutional IPO appraised of journal policies and to link to relevant MTAs from the published paper.

Several consortia are pursuing genome-wide libraries of conditional mutant strains. These tremendous resources should largely alleviate problems with sharing valuable reagents such as mouse knockout strains (see also Nature Cell Biology 9, 993-999; 2007). 